\title{
Outage in a Cellular Network Overlaid with an Ad hoc Network: The Uplink Case
}

\author{
Arshdeep S. Kahlon, Shalini Periyalwar, Halim Yanikomeroglu, Sebastian S. Szyszkowicz \\ Department of Systems and Computer Engineering, Carleton University, Ottawa, Ontario, Canada \\ Email: \{akahlon, shalinip, halim, sz\}@ sce.carleton.ca
}

\begin{abstract}
We analyze the uplink outage probability at the randomly, but not necessarily independently nor homogeneously, distributed cellular network receivers caused by an overlaid secondary ad hoc network. Unlike the previous works that assumed exact circular exclusion regions (or guard zones), we incorporate a sensing mechanism to decrease the significant effect of the nearby interferers. We consider Rayleigh-faded links, large-scale power control, and the knowledge of the initial outages in the absence of the secondary network. We derive an upper bound on the outage probability and show it to be tight when the co-channel primary receivers are separated by relatively significant distances, which is inherently the case for the cellular network. Furthermore, we derive a closed-form expression that shows the significant impact of the sensing mechanism on the spectrum sharing gains.. Additionally, we optimize the decision threshold, which is used by secondary network users to decide whether to transmit or not, in order to maximize the spectrum sharing gains under the given outage constraints.
\end{abstract}

Index Terms-Spectrum sharing, outage, secondary/cognitive network, point process, sensing, exclusion region.

\section{INTRODUCTION}

Owing to the spectrum scarcity and the inefficient spectrum use, some spectrum sharing scenarios have been discussed in the literature, where a secondary network shares the spectrum of a licensed or primary network. In the underlay concept [1], based on the fact that there is some outage tolerance available at the primary network, the secondary network is allowed to share the spectrum under the outage probability constraint given by the primary network. The investigation of outage probability at a primary receiver $(\mathrm{PR})$ requires the study of the total unwanted signal power (interference as well as noise) that it experiences.

A significant amount of literature focuses on scenarios where the primary user's spectrum is accessed by a single secondary user, as seen from the references in [2]. However, it becomes extremely challenging to extend the deterministic approach used for a few nodes towards large networks (as in the case of femtocells and ad hoc networks), where there is uncertainty as to the number and positions of the nodes. In this case, point process theory [3] becomes the preferred mathematical framework in which to study the statistical behaviour of the network. The secondary transmitters (STs) are often modeled as the points of a homogeneous Poisson point process (HPPP) on a two-dimensional plane, owing to the complete randomness of this process [4]. Most previous works focused on scenarios where the STs are deployed around a typical primary receiver (PR) of study. The outage probability at the PR as a function of the ST density has been studied in the case of a single PR in [5]-[9]. In most of these works, only the interference coming from the secondary network was considered, which might not be realistic in an interference-limited cellular network; indeed, even in the absence of the secondary network, the PR already has some outage probability due to its own primary network. In some works, an exact exclusion region (also commonly referred to as guard zone or protection zone) has been assumed around this typical PR, in order to reduce the significant effect of the nearby STs [6], [10], [11]. In reality, guaranteeing an exact circular exclusion region is impractical in a fading environment.

In many cases [12]-[14], where multiple PRs are considered, the primary network is assumed to be distributed as a Poisson point process (PPP), which can be quite a narrow assumption for a cellular network. For secondary ad hoc networks, the STs might be assumed to be distributed as PPP [3]; there are however, in general, many complex ways in which the primary nodes in the cellular network may be distributed. Moreover, there is always an inherent separation distance between co-channel devices, particularly in cellular networks, and hence the primary network cannot be accurately modeled as a PPP. For instance, while multiple PRs are taken into account in [12], the cellular BSs and users are again assumed to be distributed as a PPP. Additionally, in these works the PRs are assumed to have identical system parameters and outage probability requirements, which does not accommodate diverse primary user services.

A sensing mechanism has previously been used mostly in the overlay scheme to detect the presence of the PR [1]; an exception is [5], where the outage probability is studied at the single PR by incorporating sensing with the STs homogeneously distributed, using a lognormal fit for the interference power distribution. In [8], a sensing mechanism has been considered to detect the presence of the PR under the constraint on the outage probability given by a single PR. However, in this work, sensing of the PR by every ST is taken independently of its distance from the PR.

In this paper, we extend our analysis of the outage probability at a single PR [11] to the case of independently, but not necessarily independently nor homogeneously, distributed cellular PRs. We make use of the sensing mechanism, rather than the assumption of an exact circular exclusion region, to 
decrease the significant effect of the nearby STs, and accordingly derive an upper bound for the outage probability at the PRs. This bound is tight when the co-channel PRs are spatially separated by relatively large distances, which is the case in cellular networks. The sensing mechanism is shown to be quite significant in decreasing the outage probability at the PRs. To achieve maximum spectrum sharing gains, we optimize the decision threshold used by the STs to decide whether to transmit or not, given the outage probability constraints. Additionally, in our study, every co-channel primary signal link may have different system parameters. We confirm the analytical results derived in this paper through Monte Carlo simulations.

\section{Problem Statement}

Consider a setup where the secondary ad hoc network is overlaid on the primary cellular network as shown in Fig. 1. Let the locations of these co-channel primary receivers or base-stations (BSs) be distributed randomly over $S \subseteq \mathbb{R}^{2}$, represented by the points of a point process $\Pi_{p}=\left\{Y_{1}, Y_{2}, \ldots\right\}$. $\Pi_{p}$ can follow any statistical distribution. The secondary transmitters are assumed to be distributed as the points of a PPP $\Pi_{\mathrm{s}}=\left\{X_{1}, X_{2}, \ldots\right\}$ over $\mathbb{R}^{2}$ with density $\lambda$, and each has transmit power $\rho_{\mathrm{t}}$. We assume the location $Y_{i}$ of the $i^{\text {th }}$ PR of study to be at the origin in the polar coordinate system. Accordingly, we define a new point process $\Pi_{i, \mathrm{p}}=\left\{Y_{1, i}, Y_{2, i}, \ldots\right\} \subset \mathbb{R}^{2}$, conditioned on the location of the $i^{\text {th }} \mathrm{PR}$, where $Y_{k, i}=Y_{k}-Y_{i}$ denotes the location of the $k^{\text {th }} \mathrm{PR}$ with respect to the $i^{\text {th }} \mathrm{PR}$.

We study the outage at the $i^{\text {th }}$ PR due to the interference coming from its own (primary) network as well as from the secondary network. For a given useful signal link distance $d_{i}$, the signal received at this $i^{\text {th }} \mathrm{PR}$ is given by

$$
S_{i}=\gamma_{i} \rho_{i, \mathrm{r}}\left(d_{i}\right),
$$

where $\rho_{i, \mathrm{r}}$ is the mean received power by this PR on the desired signal link and $\gamma_{i}$ is the exponentially distributed random variable representing Rayleigh fading on the desired signal link. In the case of full large-scale path loss compensation, i.e., constant large-scale received power, we have $\rho_{i, \mathrm{r}}\left(d_{i}\right)=\rho_{i, \mathrm{r}} \forall$ $i$, as defined in modern cellular standards for the uplink case. In the absence of the secondary network, let the net unwanted signal power at the $i^{\text {th }}$ PR be $I_{i, \mathrm{p}}+\eta_{i}$, accounting for both the interference $I_{i, \mathrm{p}}$ coming from the primary network, as well as for the noise power $\eta_{i}$. The corresponding initial outage probability $\varepsilon_{i}\left(d_{i}\right)$ at this $\mathrm{PR}$ is given by

$$
\varepsilon_{i}\left(d_{i}\right)=\mathcal{P}\left(\frac{S_{i}}{I_{i, \mathrm{p}}+\eta_{i}}<\beta_{i}\right),
$$

where $\beta_{i}$ is the signal-to-interference-and-noise power ratio (SINR) threshold. Now, in the presence of a secondary network, the net unwanted signal power at the $i^{\text {th }} \mathrm{PR}$ is $I_{i, \mathrm{p}}+I_{i, \mathrm{~s}}+\eta_{i}$, where $I_{i, \mathrm{~s}}$ is the interference coming from the secondary network. The corresponding total outage probability

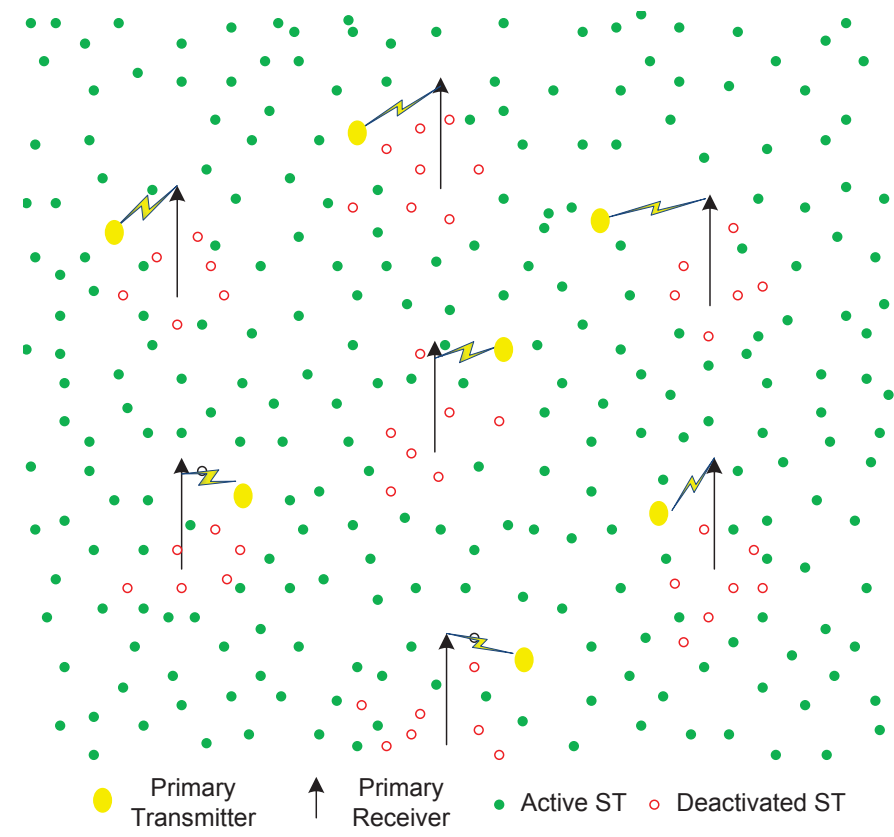

Fig. 1. Illustration of the co-channel cellular primary users sharing spectrum with the secondary ad hoc network in the uplink case.

$\varepsilon_{i, \mathrm{t}}\left(d_{i}\right)$ at this $i^{\text {th }} \mathrm{PR}$ is given by

$$
\varepsilon_{i, \mathrm{t}}\left(d_{i}\right)=\mathcal{P}\left(\frac{S_{i}}{I_{i, \mathrm{p}}+I_{i, \mathrm{~s}}+\eta_{i}}<\beta_{i}\right) .
$$

\section{Outage Analysis}

We begin by studying the outage probability at the $i^{\text {th }}$ PR due to both the secondary interference $I_{i, \mathrm{~s}}$ and to the primary interference $I_{i, \mathrm{p}}$. We use the theory of marked Poisson processes to model the STs as the points of a PPP. We define $\Pi_{i, \mathrm{~s}}=\left\{\left(X_{j, i}, \gamma_{j, i}\right) \mid X_{j} \in \Pi_{\mathrm{s}}\right\}$ over the product space $\mathbb{R}^{2} \times \mathbb{R}^{+}$, where $\gamma_{j, i}$ is the mark associated with the interferer at $X_{j, i}=X_{j}-Y_{i}$ and represents fading on the channel between this $j^{\text {th }}$ interferer and the $i^{\text {th }}$ PR. The marks are distributed identically and independently for every $j$, and independently of $X_{j, i}$. Due to the spatial stationarity of $\Pi_{\mathrm{s}}$ and from the Marking Theorem [15], $\Pi_{i, \mathrm{~s}}$ is also a PPP.

We now consider two cases: one with and the other without a sensing mechanism ${ }^{1}$. In the first case, there is no restriction on how close the STs can be to the PR, while in the second case, a sensing mechanism is used to decrease the significant effect of the nearby interfering STs by deactivating the nearby STs.

\section{A. Without Sensing Mechanism}

In this case, there is no sensing mechanism adopted by the STs to deactivate the dominant STs near the PRs. Due to the

\footnotetext{
${ }^{1}$ Note that the sensing mechanism employed here is used to estimate the closeness of the ST to the PR, so that the nearby STs are most likely to be deactivated. This is different from the commonly used sensing mechanism in the overlay scheme [2] of spectrum sharing, where sensing is used to detect the presence or the absence of the PR or PRs.
} 
spatial stationarity property of the HPPP [15], the statistics of the interference coming from the STs at any point are the same, therefore $I_{i, \mathrm{~s}}=I_{\mathrm{s}} \forall Y_{i} \in \Pi_{\mathrm{p}}$. Moreover, $I_{i, \mathrm{~s}}$ and $I_{i, \mathrm{p}}$ are independent, and it follows that we can apply the outage probability equation derived for a single PR [11, Eq. 10] to the two independent unwanted signal sets, in order to find the total outage probability:

$$
\varepsilon_{i, \mathrm{t}}\left(d_{i}\right)=1-\left(1-\varepsilon_{i}\left(d_{i}\right)\right) \exp \left(\frac{-\lambda 2 \pi^{2}\left(\kappa_{i} \rho_{\mathrm{t}}\right)^{2 / \alpha}}{\alpha \sin (2 \pi / \alpha)}\right),
$$

where $\kappa_{i}=\beta_{i} / \rho_{i, \mathrm{r}}$, and $\alpha$ is the path loss exponent.

Let $\varepsilon_{i, \mathrm{t}}=\mathbb{E}_{d_{i}}\left(\varepsilon_{i, \mathrm{t}}\left(d_{i}\right)\right)$ and $\varepsilon_{i}=\mathbb{E}_{d_{i}}\left(\varepsilon_{i}\left(d_{i}\right)\right)$; then, from (4), we obtain

$$
\varepsilon_{i, \mathrm{t}}=1-\left(1-\varepsilon_{i}\right) \exp \left(\frac{-\lambda 2 \pi^{2}\left(\kappa_{i} \rho_{\mathrm{t}}\right)^{2 / \alpha}}{\alpha \sin (2 \pi / \alpha)}\right),
$$

under large-scale power control.

\section{B. With Sensing Mechanism}

The sensing mechanism is used in order to avoid nearby secondary transmissions around each PR. Distributed sensing is considered for all the PRs, such that each ST senses and decides whether to transmit independently of all the other STs. The ST senses a beacon broadcast by the PR on an out-of-band channel, assuming a full-duplex channel for both the PRs and the STs. The sensing and data channels are independent of each other. Alternatively, sensors can be deployed at the STs to perform the sensing operations. Some higher layer sensing protocols can be incorporated under the given framework, but this is outside the scope of this study, which is focused on statistical aspects only. More information about distributed sensing can be obtained from [2] and the references therein. We assume that the detection of the PR by any ST is independent of the beacons transmitted by the other PRs, since these are generally low power beacons transmitted by distant PRs. In other words, the sensing channel is assumed not to suffer from interference.

We define a set of deactivated STs that are not allowed to transmit around the $k^{\text {th }} \mathrm{PR}$ (located at $Y_{k, i}$ ) as

$$
\begin{array}{r}
\mathbb{S}_{k, i}=\left\{\left(X_{j, i}, \gamma_{j, i}\right): \frac{\gamma_{j, k, i}^{\prime} \rho_{\mathrm{b}}}{\eta\left|X_{j, i}-Y_{k, i}\right|^{\alpha}}>\beta_{i, \mathrm{th}},\right. \\
\left.\left(X_{j, i}, \gamma_{j, i}\right) \in \Pi_{i, \mathrm{~s}}, Y_{k, i} \in \Pi_{i, \mathrm{p}}\right\},
\end{array}
$$

where $\gamma_{j, k, i}^{\prime}$ is the exponentially distributed random variable representing Rayleigh fading on the sensing channel between the $k^{\text {th }} \mathrm{PR}$ and the $j^{\text {th }} \mathrm{ST}$, and is independent of $\gamma_{j, i}$. $\rho_{\mathrm{b}}$ is the beacon power, $\beta_{i, \text { th }}$ the decision threshold for transmission based on the signal-to-noise power ratio (SNR), and $\eta$ the background noise power. Also, in the case of energy-based detection [2], which is a function of SNR, an equivalent $\beta_{\mathrm{th}}$ can be incorporated.

Hence, the set of STs that are allowed transmission can be written

$$
\Pi_{i, \mathrm{~s}, \mathrm{t}}=\Pi_{i, \mathrm{p}} \backslash \bigcup_{Y_{k, i} \in \Pi_{i, \mathrm{p}}} \mathbb{S}_{k, i}
$$

STs that receive the beacon (transmitted by the PRs) above a certain threshold level are not allowed to transmit, as there are high chances that they are close to a PR. The probability of an ST being allowed to transmit is dependent on the positions of the PRs. Since the PRs follow any general point process, where the PRs might not be independent of each other, we can say that the corresponding primary transmitters are also not independent of each other. Therefore, the net interference powers $I_{i, \mathrm{p}}$ and $I_{i, \mathrm{~s}}$ coming from the two networks are not independent. Due to this interaction between the PRs and the STs, and consequently between the interfering primary transmitters and the STs, it seems infeasible to obtain the exact outage probability expressions, which was suggested in [3]. We may however make use of the facts that 1) the secondary network generally has a high density of nodes and short transmission ranges and 2) the cellular co-channel PRs (particularly in the uplink case) are inherently separated by relatively large distances in the cellular network. This allows us to derive an upper bound for the outage probability at the PRs. This bound is tight and helps us obtain analytical insights on the effect of various system parameters.

It is easy to show that $I_{i, \mathrm{~s}}^{+} \geq I_{i, \mathrm{~s}}$, where $I_{i, \mathrm{~s}}^{+}$is the interference coming from the STs distributed as $\Pi_{i, \mathrm{~s}, \mathrm{t}}^{\prime}=$ $\Pi_{i, \mathrm{~s}} \backslash \mathbb{S}_{i, i}$ and $I_{i, \mathrm{~s}}$ is the interference coming from $\Pi_{i, \mathrm{~s}, \mathrm{t}}=$ $\Pi_{i, \mathrm{~s}} \backslash \bigcup_{Y_{k, i} \in \Pi_{i, \mathrm{p}}} \mathbb{S}_{k, i}$. Now, since $\varepsilon_{i, \mathrm{t}}^{+}\left(d_{i}\right)$ is the total outage probability due to $I_{i, \mathrm{~s}}^{+}+I_{i, \mathrm{p}}$, we can say that $\varepsilon_{i, \mathrm{t}}\left(d_{i}\right) \leq \varepsilon_{i, \mathrm{t}}^{+}\left(d_{i}\right)$.

Because $I_{i, \mathrm{p}}$ and $I_{i, \mathrm{~s}}^{(u)}$ are independent, in which case we can write $\varepsilon_{i}^{+}\left(d_{i}\right)$, as previously,

$$
\varepsilon_{i, \mathrm{t}}^{+}\left(d_{i}\right)=1-\left(1-\varepsilon_{i}\left(d_{i}\right)\right) \mathcal{L}_{I_{i, \mathrm{~s}}^{+}}\left(\kappa_{i}\right) .
$$

Now $\Pi_{i, \mathrm{~s}, \mathrm{t}}^{\prime}=\Pi_{i, \mathrm{~s}} \backslash \mathbb{S}_{i, i}$ is formed by a thinning operation (in our context, deactivating the STs) on $\Pi_{i, \mathrm{~s}}$ [15], where the probablity of any ST getting thinned depends on the SNR on the sensing channel, which is independent of the other sensing channels. Hence, $\Pi_{i, \mathrm{~s}, \mathrm{t}}^{\prime}$ also forms a PPP [15], but with a nonuniform density given by

$$
\lambda(r, \theta)=\lambda \mathcal{P}\left(\frac{\gamma_{j, i}^{\prime} \rho_{\mathrm{b}}}{\eta r^{\alpha}}<\beta_{i, \mathrm{th}}\right)=\lambda\left(1-e^{-\eta r^{\alpha} \beta_{i, \mathrm{th}} / \rho_{\mathrm{b}}}\right) .
$$

We now use the Campbell's theorem for marked Poisson processes [15] to incorporate the sensing mechanism, and it follows that

$$
\begin{aligned}
\mathcal{L}_{I_{i, \mathrm{~s}}^{+}}\left(\kappa_{i}\right)=\exp & \left\{\lambda \int_{0}^{2 \pi} \int_{0}^{\infty} \int_{0}^{\infty}\left(e^{-y \kappa_{i} \rho_{\mathrm{t}} r^{-\alpha}}-1\right)\right. \\
& \left.\cdot\left(1-e^{-\eta r^{\alpha} \beta_{i, \mathrm{th}} / \rho_{\mathrm{b}}}\right) r \mathrm{~d} y \mathrm{~d} r \mathrm{~d} \theta\right\},
\end{aligned}
$$

Substituting $x=r^{\alpha}$ in (10) and using (3.193.4) and (3.383.10) of [16], we derive the closed-form result

$$
\mathcal{L}_{I_{i, \mathrm{~s}}^{+}}\left(\kappa_{i}\right)=\exp \left(-\lambda \Omega_{i}\left(\beta_{i, \mathrm{th}}\right)\right),
$$


where

$$
\begin{gathered}
\Omega_{i}\left(\beta_{i, \mathrm{th}}\right)=\frac{2 \pi}{\alpha}\left(\kappa_{i} \rho_{\mathrm{t}}\right)^{2 / \alpha}\left(\frac{\pi}{\sin (2 \pi / \alpha)}\right. \\
\left.-\exp \left(\frac{\kappa_{i} \rho_{\mathrm{t}} \beta_{i, \mathrm{th}} \eta}{\rho_{\mathrm{b}}}\right) \Gamma\left(\frac{2}{\alpha}\right) \Gamma\left(1-\frac{2}{\alpha}, \frac{\kappa_{i} \rho_{\mathrm{t}} \beta_{i, \mathrm{th}} \eta}{\rho_{\mathrm{b}}}\right)\right)
\end{gathered}
$$

Therefore, the upper bound $\varepsilon_{i, \mathrm{t}}^{+}\left(d_{i}\right)$ on the total outage probability at the $i^{\text {th }}$ PR is given by

$$
\varepsilon_{i, \mathrm{t}}^{+}\left(d_{i}\right)=1-\left(1-\varepsilon_{i}\left(d_{i}\right)\right) \exp \left(-\lambda \Omega_{i}\left(\beta_{i, \mathrm{th}}\right)\right) .
$$

Now, if $\varepsilon_{i}^{+}=\mathbb{E}_{d_{i}}\left(\varepsilon_{i}^{+}\left(d_{i}\right)\right)$ and $\varepsilon_{i}=\mathbb{E}_{d_{i}}\left(\varepsilon_{i}\left(d_{i}\right)\right)$, we can express the upper bound $\varepsilon_{i, \mathrm{t}}^{+}$as

$$
\varepsilon_{i, \mathrm{t}}^{+}=1-\left(1-\varepsilon_{i}\right) \exp \left(-\lambda \Omega_{i}\left(\beta_{i, \mathrm{th}}\right)\right),
$$

under large-scale power control.

The outage probability result obtained in the case of the sensing mechanism can be thought of as pessimistic; however, it is also quite safe, as sensing errors can occur due to hardware inaccuracies [2]. The upper bound derived here gives assurance that even if some sensing errors do occur, the primary services do not suffer beyond the outage probability constraint specified by the PRs.

We now want to find the optimum decision threshold $\beta_{i \text {,th }}^{\text {opt }}$ for which the maximum spectrum sharing gains (here, the number of STs) are achieved, while satisfying the given outage probability constraint $\varepsilon_{i, \text { th }}$ at every primary receiver, located at $Y_{i} \in \Pi_{\mathrm{p}}$.

In order to ensure that the outage probability condition is satisfied, we impose $\varepsilon_{i, \mathrm{t}}^{+} \leq \varepsilon_{i, \mathrm{th}}$. Equivalently, from (14), we can write this condition as

$$
\beta_{i, \mathrm{th}} \leq \Omega_{i}^{-1}\left(-\frac{1}{\lambda} \log \left(\frac{1-\varepsilon_{i, \mathrm{th}}}{1-\varepsilon_{i}}\right)\right) .
$$

From (10), or from differentiating (12), it can easily be shown that $\Omega_{i}\left(\beta_{i, \mathrm{th}}\right)$ is an increasing function of $\beta_{i, \mathrm{th}}$. We can therefore write the above equation as

$$
\beta_{i, \mathrm{th}} \leq \Omega_{i}^{-1}\left(-\frac{1}{\lambda} \log \left(\frac{1-\varepsilon_{i, \mathrm{th}}}{1-\varepsilon_{i}}\right)\right) .
$$

Using (7), the total number of active STs is

$$
\left|\Pi_{i, \mathrm{~s}, \mathrm{t}}\right|=\left|\Pi_{i, \mathrm{~s}}\right|-\left|\bigcup_{Y_{k, i} \in \Pi_{i, \mathrm{p}}} \mathbb{S}_{k, i}\right|,
$$

where $|\mathcal{S}|$ denotes the cardinality of any set $\mathcal{S}$. In the case of the cellular primary network, the PRs are spatially separated by relatively larger distances, and therefore the deactivation of an ST is most likely due to the sensing of the nearest PR only, as depicted in Fig. 1. We may therefore write

$$
\begin{aligned}
& \left|\Pi_{i, \mathrm{~s}, \mathrm{t}}\right| \gtrsim\left|\Pi_{i, \mathrm{~s}}\right|-\sum_{Y_{k, i} \in \Pi_{i, \mathrm{p}}}\left|\mathbb{S}_{k, i}\right| \\
= & \left|\Pi_{i, \mathrm{~s}}\right|-\sum_{\substack{\left(X_{j, i}, \gamma_{j, i}\right) \in \Pi_{i, \mathrm{~s}} \\
Y_{k, i} \in \Pi_{i, \mathrm{p}}}} u\left(\frac{\gamma_{j, k, i}^{\prime} \rho_{\mathrm{b}}}{\eta\left|X_{j, i}-Y_{k, i}\right|^{\alpha}}-\beta_{i, \mathrm{th}}\right),
\end{aligned}
$$

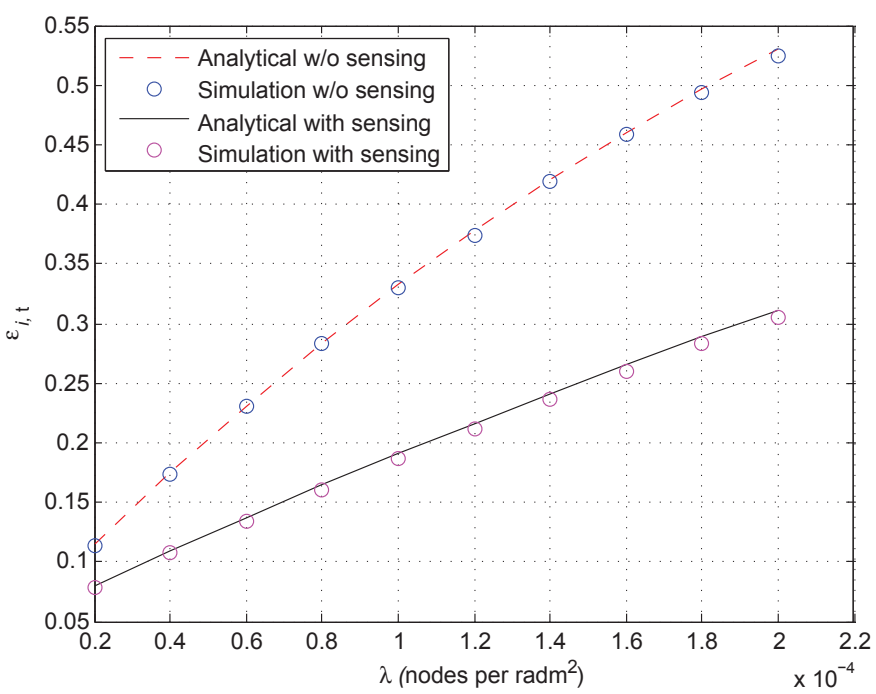

Fig. 2. Total outage probability $\varepsilon_{i, \mathrm{t}}$ at the $i^{\text {th }} \mathrm{PR}$ with the density of STs being $\lambda$ for $\varepsilon_{i}=0.05, \alpha=3, \rho_{i, \mathrm{r}}=1 \mu \mathrm{W}, \beta_{i}=10, \rho_{\mathrm{t}}=1 \mathrm{~mW}, \rho_{\mathrm{b}}=1 \mathrm{~W}$, and $\beta_{i, \mathrm{th}}=10$. The distance between adjacent co-channel BSs is taken as $500 \mathrm{~m}$.

where $u(x)$ is equal to 1 for $x>0$, and to zero otherwise. We then see that, for any realisation of channels and node positions, the number of active STs is always maximized by maximizing every $\beta_{i, \mathrm{th}}$. Therefore, from (16), the optimum sensing thresholds are given by

$$
\beta_{i, \mathrm{th}}^{\mathrm{opt}}=\Omega_{i}^{-1}\left(-\frac{1}{\lambda} \log \left(\frac{1-\varepsilon_{i, \mathrm{th}}}{1-\varepsilon_{i}}\right)\right), \forall Y_{i} \in \Pi_{\mathrm{p}} .
$$

\section{Numerical Results}

In order to show the effect of using a sensing mechanism on the outage probability at the PR, and to validate the analytical results (5) and (14) against simulation, we plot in Fig. 2 the total outage probability $\varepsilon_{i, \mathrm{t}}$ at the $i^{\text {th }} \mathrm{PR}$ against the density of interferers (here, STs) $\lambda$, with the following simulation paramaters: $\varepsilon_{i}=0.05, \alpha=3, \rho_{i, \mathrm{r}}=1 \mu \mathrm{W}, \beta_{i}=10, \rho_{\mathrm{t}}=1 \mathrm{~mW} \forall$ $Y_{i} \in \Pi_{\mathrm{p}}$, and $\beta_{i, \mathrm{th}}=10$. To perform the worst-case analysis when the sensing mechanism is present, we consider the tier of co-channel PRs (or BSs) around the $i^{\text {th }} \mathrm{PR}$ at a distance of only $500 \mathrm{~m}$ from the $i^{\text {th }} \mathrm{PR}$, and assume a quite high probe power $\rho_{\mathrm{b}}=1 \mathrm{~W}$. As done in [11], we simulate the primary interference plus noise power $\left(I_{i, \mathrm{p}}+\eta_{i}\right)$ : its distribution is assumed to be gamma with scale parameter 2 . We may in fact take any other distribution to model the sum of the primary network interference and noise, since the results are only dependent on the initial outage probability $\varepsilon_{i}$. We tune the shape parameter of the gamma distribution to obtain any given initial outage probability $\varepsilon_{i}$ of study.

We observe that the upper bound on the outage probability derived in the case of sensing is quite tight with the simulation curve. This bound should be tighter in the actual cellular systems, where the PRs are inherently separated by relatively larger distances. Furthermore, we observe from Fig. 2 that there is a significant decrease in the total outage probability suffered by the PR when the density of the STs increases, as 


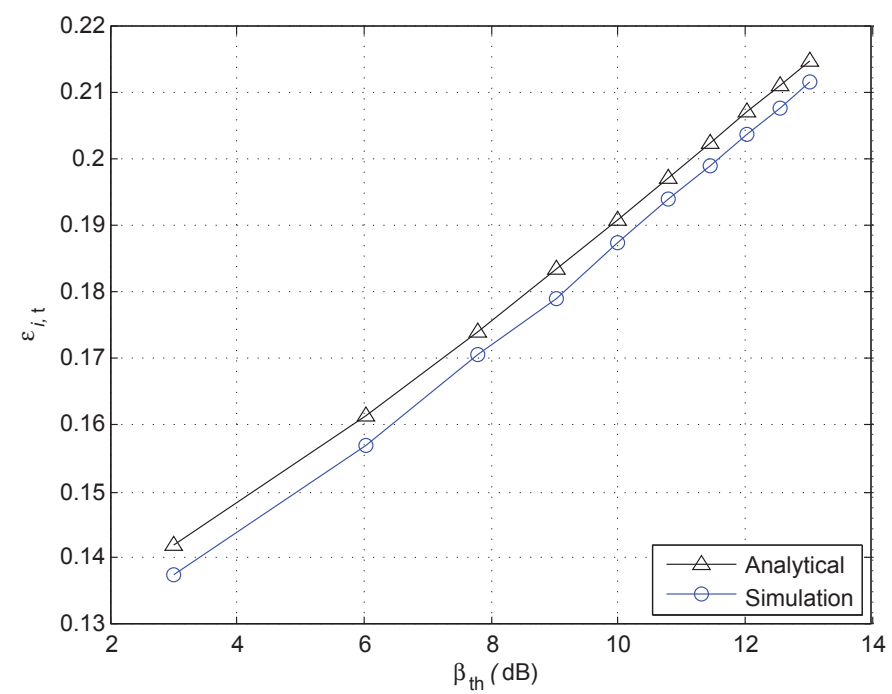

Fig. 3. Total outage probability $\varepsilon_{i, \mathrm{t}}$ at the $i^{\mathrm{th}} \mathrm{PR}$ with the decision threshold $\beta_{i, \mathrm{th}}=\beta_{\mathrm{th}}$, for $\lambda=10^{-4} / \mathrm{m}^{2}, \varepsilon_{i}=0.05, \alpha=3, \rho_{i, \mathrm{r}}=1 \mu \mathrm{W}, \beta_{i}=10, \rho_{\mathrm{b}}=1$ $\mathrm{W}, \eta=10^{-6}$, and $\rho_{\mathrm{t}}=1 \mathrm{~mW}$. The distance between adjacent BSs is taken as $500 \mathrm{~m}$.

well as when the sensing mechanism is used to alleviate the severe effect of nearby interfering transmissions. In Fig. 3, we show the effect of the decision threshold on the outage for the same simulation parameters as above, while fixing $\lambda=10^{-4} / \mathrm{m}^{2}$. The derived upper bound on the outage probability using (14) is observed to be tight with the simulation curve.

More generally, using the results derived in this paper, we could analyze the effect other system parameters (ST transmit power, initial outage probability, path loss exponent, etc.) under the given framework.

\section{CONCLUSION}

We considered the uplink case of a spectrum sharing scenario where a secondary ad hoc network is overlaid on the randomly, but not necessarily independently nor homogeneously, distributed co-channel PRs in the cellular network. We analyzed the outage probability experienced by the cellular receiver nodes, with and without a sensing mechanism. Although the spectrum sharing between the primary cellular network with a secondary ad hoc network has been considered before, to the best of the authors' knowledge, we have derived the closed form expression for the outage probability incorporating both sensing and random distribution of nodes for the first time. We made use of the fact that the PRs (or BSs) are inherently separated by relatively large distances, in order to derive a tight upper bound on the outage probability in the case when a sensing mechanism is present. We showed a significant decrease in the outage probability at the PR when the sensing mechanism was used to avoid the significant effect of the nearby secondary network transmissions. In order to maximize the spectrum sharing gains under some given outage probability constraints, we optimized the transmission decision threshold used by the secondary users. It is worth mentioning that the results derived are applicable to any set of interferers (from the same or from a different network) distributed around any receiver of study.

\section{ACKNOWLEDGEMENT}

The authors would like to thank Dr. Muhammad Aljuaid for his helpful comments.

\section{REFERENCES}

[1] Q. Zhao and B. Sadler, "A survey of dynamic spectrum access," IEEE Signal Process. Mag., vol. 24, no. 3, pp. 79-89, May 2007.

[2] T. Yucek and H. Arslan, "A survey of spectrum sensing algorithms for cognitive radio applications," IEEE Commun. Surveys Tuts., vol. 11, no. 1, pp. 116-130, Mar. 2009.

[3] J. Andrews, R. Ganti, M. Haenggi, N. Jindal, and S. Weber, "A primer on spatial modeling and analysis in wireless networks," IEEE Commun. Mag., vol. 48, no. 11, pp. 156-163, Nov. 2010.

[4] M. Win, P. Pinto, and L. Shepp, "A mathematical theory of network interference and its applications," Proc. IEEE, vol. 97, pp. 205-230, Feb. 2009.

[5] A. Ghasemi and E. Sousa, "Interference aggregation in spectrum-sensing cognitive wireless networks," IEEE J. Sel. Topics Signal Process., vol. 2, no. 1, pp. 41-56, Feb. 2008.

[6] R. Menon, R. Buehrer, and J. Reed, "On the impact of dynamic spectrum sharing techniques on legacy radio systems," IEEE Trans. Wireless Commun., vol. 7, no. 11, pp. 4198-4207, Nov. 2008.

[7] V. Mordachev and S. Loyka, "On node density - outage probability tradeoff in wireless networks," IEEE J. Sel. Areas Commun., vol. 27, no. 7, pp. 1120-1131, Sept. 2009.

[8] M. Khoshkholgh, K. Navaie, and H. Yanikomeroglu, "Impact of the secondary network on the outage performance of the primary service in spectrum sharing," in IEEE International Conference on Communications, May 2010.

[9] R. Dahama, K. Sowerby, and G. Rowe, "Outage probability estimation for licensed systems in the presence of cognitive radio interference," in IEEE Vehicular Technology Conference, Apr. 2009.

[10] M. Vu, N. Devroye, and V. Tarokh, "On the primary exclusive region of cognitive networks," IEEE Trans. Wireless Commun., vol. 8, no. 7, pp. 3380-3385, July 2009.

[11] A. S. Kahlon, S. S. Szyszkowicz, S. Periyalwar, and H. Yanikomeroglu, "Outage due to an independent unwanted signal under Rayleigh fading and its application in spectrum sharing," submitted to IEEE Trans. Wireless Commun., Apr. 2011.

[12] K. Huang, V. Lau, and Y. Chen, "Spectrum sharing between cellular and mobile ad hoc networks: transmission-capacity trade-off," IEEE J. Sel. Areas Commun., vol. 27, no. 7, pp. 1256-1267, Sept. 2009.

[13] C. Yin, C. Chen, T. Liu, and S. Cui, "Generalized results of transmission capacities for overlaid wireless networks," in IEEE International Symposium on Information Theory, June 2009, pp. 1774-1778.

[14] C. Lee and M. Haenggi, "Interference and outage in doubly Poisson cognitive networks," in Proc. International Conference on Computer Communications and Networks, Aug. 2010.

[15] D. Stoyan, W. Kendell, and J. Mecke, Stochastic Geometry and it Applications, 2nd ed., ser. Wiley Series in Probability and Mathematical Statistics. New York: Wiley, 1995.

[16] I. S. Gradshteyn and I. M. Ryzhik, Tables of Integrals, Series, and Products, 7th ed. Academic Press, 2007. 